\title{
Parametric analysis on a simple design water reaction turbine for low-head low-flow Pico-hydro generation system
}

\author{
N. A. M. Rais ${ }^{1}$ and M. F. Basar ${ }^{1}$ \\ ${ }^{1}$ Faculty of Electrical and Electronic Engineering Technology, Universiti Teknikal Malaysia Melaka, 76100 Durian Tunggal, Melaka, Malaysia \\ Phone: +6062701000; Fax: +6062701022
}

ABSTRACT - This paper presents a parametric analysis of the outward flow reaction type turbine known as a Z-Blade turbine for low-head low-flow conditions. By applying the principles of mass conservation, momentum and energy, a nomogram was designed to investigate the theoretical performance characteristics. Based on the parametric analysis and the governing equations and experimental results, attempts have been made to prove that the mass flow rate, angular speed, centrifugal head, power output and efficiency respond dynamically to the water head, radius of the rotor, size of the PVC pipes and the nozzle exit area. A turbine with a 1" pipe diameter gives a higher performance compared to a $1 / 2$ " pipe diameter, and certainly the performances of both pipe sizes are improved when the supplied potential energy is increased. This innovative turbine has a maximum rotational speed at an optimum turbine diameter at $0.6 \mathrm{~m}$, accompanied by a point where there is a sudden reduction in the water flow rate, where previously the increment in the water flow rate was very high. This can shows from the outcome nomogram with 1" pipe diameter can perform $350 \mathrm{rpm}$ speed with $1.48 \mathrm{~L} / \mathrm{sec}$ water flow. The Z-Blade turbine has been examined and has shown good potential to be used for low-head ( $3 \mathrm{~m}, 4 \mathrm{~m}$ and $5 \mathrm{~m})$ and low-flow (less than $2.5 \mathrm{~L} / \mathrm{sec}$ ) conditions.
ARTICLE HISTORY

Received: $13^{\text {th }}$ Aug 2020

Revised: $5^{\text {th }}$ Feb 2021

Accepted: 25 $5^{\text {th }}$ Feb 2021

\section{KEYWORDS}

Low head;

low flow;

parametric;

pico-hydro;

reaction turbine;

Z-Blade.

\section{INTRODUCTION}

Recently, many countries have been facing fluctuations in the water flow rate, where the mass flow rate and waterhead have been disrupted due to dry spells. It is a time of minimum power production, and a key challenge of a pico-hydro power plant is to maintain the performance of its generation system on a regular basis. The limited power source is mainly 'run of river', so a dam system does not require, but instead a limited barrier called small lake can be installed to regulate and maintain flow of water. In this particular system, the head and the flow are two essential components. The head variable refers to the pressure of water that defines the vertical fall of water. The water intake and turbine also establish an altitude [1-3]. ost turbines that have been studied and commercialized so far are only suitable for operating under highhead high-flow, low-head high-flow and high-flow low-head [4, 5] conditions. There are many low-head low-flow areas that are not being utilized for power generation [6] due to the lack of hydro technology [6]. In this research, an innovative reaction type turbine called a Z-blade turbine will be the precursor in this area that is often underestimated and neglected by many people.

This research work was inspired by the cross pipe turbine (CPT) and split reaction water turbine (SRT), where both turbines are categorized as simple reaction turbines. CPT and SRT are the result of efforts that were made to grab the opportunities available in an area that is not widely discussed and explored. CPT, which was developed using standard galvanized iron (GI) pipe fittings, was discussed in detail by Date et al. [7, 8]. Interestingly, the SRT was manufactured after its inventor had experimented and declared that the CPT was not suitable for low-head hydro applications. The SRT is less complex in its geometrical design compared to other reaction hydraulic turbines. The SRT, with its improved diskshaped rotor design, has been proven to be capable of operating in low-head conditions. With a rotor diameter of 0.122 $\mathrm{m}$, it is capable of producing 150 Watts of electrical power at $40 \mathrm{kPa}$ with a water flow rate consumption of about 20 L/sec [8-10]. The disadvantage of the SRT is that it needs a high water flow rate even though it can be operated in lowhead water conditions.

This paper introduces an innovative Z-blade turbine which addresses operational problems at low-head low-flow water resources. The performance characteristics of the Z-blade turbine were explored based on the parametric analysis that was performed via the governing equations by applying the principles of mass conservation, momentum, and energy. This paper also presents a designed nomogram in order to show the relationship between the parameters involved in determining the performance of a turbine. Furthermore, the nomogram is able to predict the overall turbine system immediately with only one given parameter.

The outward-flow reaction type turbine proposed in this research closely resembles the CPT in its geometrical design, but it has been modified and upgraded. The Z-blade turbine introduced in this paper has been tested under low-head ( $3 \mathrm{~m}$ to $5 \mathrm{~m})$ and extremely low mass flow rate $(\leq 2.5 \mathrm{~kg} / \mathrm{sec})$ conditions. This turbine works on a similar concept as that of the 
water sprinkler. It can be easily fabricated, and requires no high-tech manufacturing machinery and expert workers due to its non-complex design. The turbine blades are made using standard PVC plumbing pipes and fittings, making it an inexpensive reaction machine. The turbine is developed based on experimental investigations and parametric analyses of the SRT and CPT, as reported by [6-10].

There are fifteen parameters that have to be analysed in order to understand the behaviour of this hydro machine based on the experimental data. Predominant parameters, such as the angular speed $(\omega)$, mass flow rate $(\dot{m})$, optimum turbine

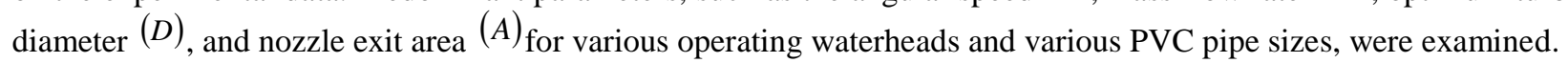

\section{THE Z-BLADE TURBINE'S PARAMETRIAL STUDY}

The paper aims to suggest optimal parameters to calculate the maximum turbine output on the basis of test data and theoretical comparisons. The parametric analysis of the experimental data and theoretical prediction under specified conditions are investigated based on governing analytical equations. The governing equations were developed from the principles of conservation of mass, momentum, and energy [8-10]. And addition, this paper will demonstrate that the innovative turbine has a great potential to be implmented in a pico-hydro system, especially at low-head and low-flow water supplies, similar to the application of a garden water sprinkler.

The Z-blade proposed in this paper works on the same concept as a water sprinkler, which is an example of a reaction machine which is widely used today for watering plants. This Z-blade also refers to other reaction turbines that function on the same concept as discussed in previous researches [2, 3, 6]. Generally, the reaction type turbine operates with pressurized water entering the turbine vertically and exiting tangentially. However, some assumptions have to be made in the analysis [8-10]. The assumptions made are that the energy cannot be dissipated through viscosity, that there is no turbulence as it is a laminar flow, and that the water is incompressible due to its constant density, unlike air, which can be compressed. The other matters that are neglected are the mechanical losses, including frictional losses in the bearings and windage losses during the rotation of the rotor.

In Figure 1, the criteria used in the Ideal Condition Analysis for evaluating the efficiency of the Z-blade turbine are shown below. These principles of an ideal condition relies on several following assumptions done by [8-10]; with constant water density, inability of energy to be dissipated through viscosity, inconsideration for any losses related to fluid flow from water tank, the mechanical losses and frictional losses of the pipe coupling. Such variables are comparable to those used for the investigations of SRT and CPT achievement under selected conditions [6-8]. Red boxes are the various parameters used to analyze the effect on the yellow box parameters. In the meantime, the green boxes are the defined parameters on the basis that acceleration is not altered due to gravity and water density is not altered due to stable temperature. Those parameters in governing equations are refer to the parameters of ideal condition.

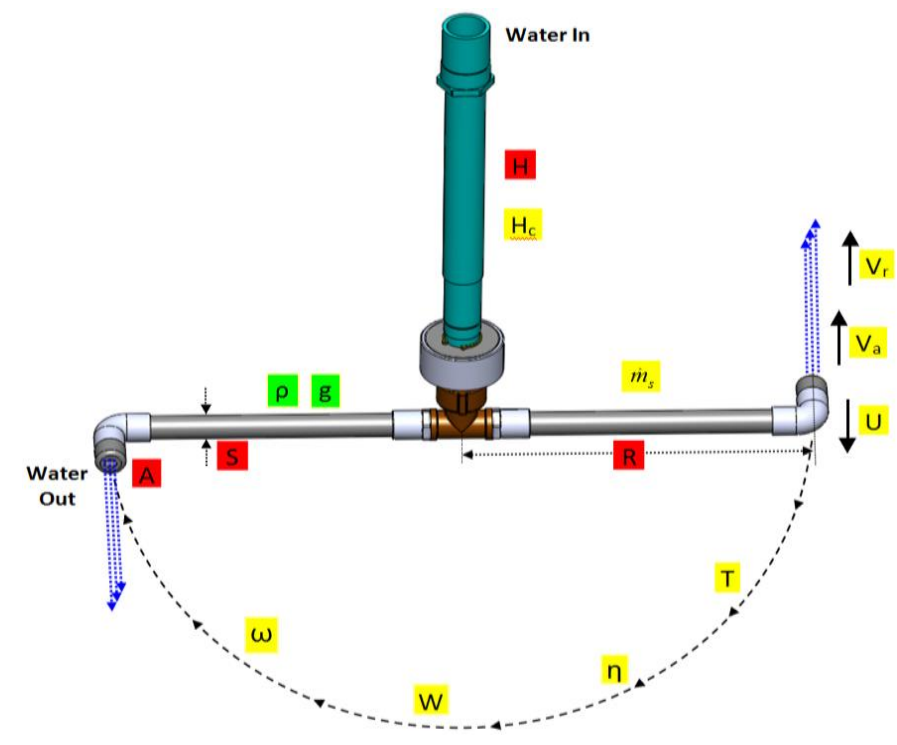

Figure 1. Rotor stationary reference frame

With the concept of preserving mass, momentum, and energy, the behavior of a Z-blade reaction water turbine under the viscous fluid water condition can be defined. The guiding equations were derived from [6, 10] in relation to Figure 1 for an ideal situation, where no frictional loss has taken place.

$$
U=R \omega
$$




$$
\begin{aligned}
V_{a} & =V_{r}-U \\
V_{a} & =V_{r}-R \omega \\
\frac{1}{2} \rho V_{r}^{2} & =\rho g\left(H+H_{c}\right)
\end{aligned}
$$

Centrifugal head $\left(\mathrm{H}_{\mathrm{c}}\right)$, when the turbine is not stationary $(\omega \neq 0)$ is given by,

$$
H_{c}=\frac{U^{2}}{2 g}=\frac{R^{2} \omega^{2}}{2 g}
$$

Mass flow rate (m), sprayed out of the nozzle can be expressed when the turbine is stationary $(\omega=0)$ is,

$$
\dot{m}_{S}=\rho A \sqrt{2 g H}
$$

The angular speed $(\omega)$ of the rotor can be calculated by rewriting Eq. (6),

$$
\omega=\sqrt{\frac{\left(\frac{\dot{m}}{\rho A}\right)^{2}-2 g H}{R^{2}}}
$$

The mechanical output power $\dot{W}$, produced by the turbine,

$$
\dot{W}=T \omega
$$

The efficiency $(\eta)$ of the system in converting potential energy to work can be written as

$$
\eta=\frac{\dot{W}}{\dot{m} g H}
$$

In experimental research, water head $(\mathrm{H})$, blade radius $(\mathrm{R})$, pipe size $(\mathrm{S})$, nozzle exit area $(\mathrm{A})$ and a mass flow rate (m) of a rotor $(\omega)$ are the parameters that may be gathered. During the meantime, Eq. (1) to Eq. (9) can be used to determine other reation turbine parameters.

\section{LABORATORY TESTING}

The turbine was provided with pumping water from the top of the blade and the rotor was installed in the bottom of the rotor with the Z-blade turbine. With this arrangement, as shown in Figure 2, it was able to benefit fully from the gravitational potential energy supplied by the water in the tank. The test rig was able to provide a waterhead of up to $5 \mathrm{~m}$ and a mass water flow rate of up to $3 \mathrm{~L} / \mathrm{sec}$. The water test rig shown in Figure 2 depicts the method used in real time hydro sites. In any real application, the pico-hydro power generation system to be implemented is similar to the water test rig shown in Figure 2. It can be located either on the river bank (run-off river) or in the streams (run-on river). The water tank will be filled with river water and the water coming out from the turbine can be channeled back into the river. 


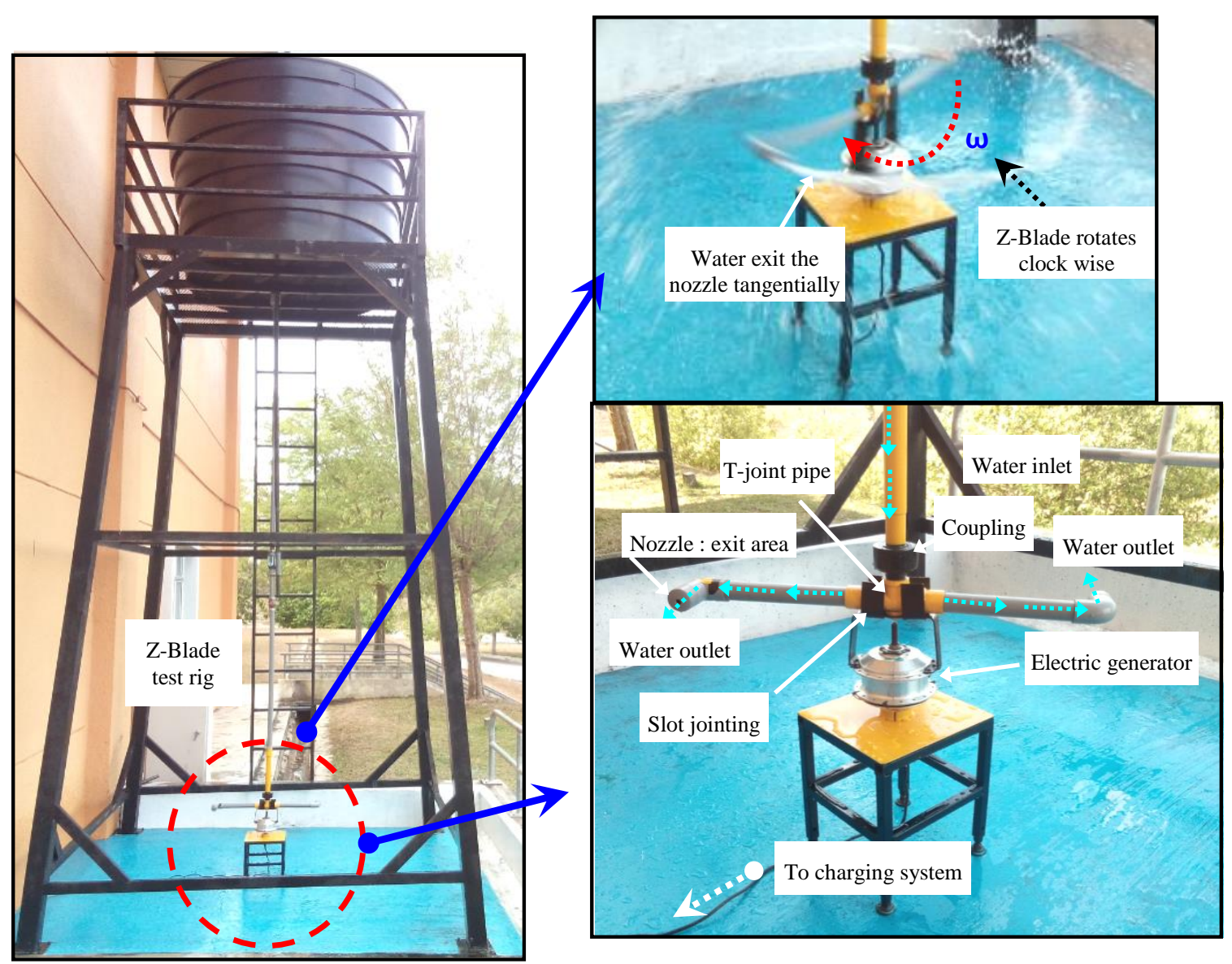

Figure 2. Z-blade turbine experimental test rig

The Class D-standard PVC grey plumbing pipes and installation of two tubes of 1/2" (nomeral diameter 15mm) and $1 "$ (nomeral diameter $25 \mathrm{~mm}$ ) have been established for the construction of this water turbine. PVC pipes have different characteristics in terms of power, ease of service, low cost and light weight transportation. In addition, this revolutionary turbine is geometrically simple and can be conveniently built without the need of expertise. The Z-blade Turbine normally rotates in the opposite direction of the water jet and produces mechanical and electrical strength at the same time as the fluid tangent out of the conduit, creating a reaction force.

\section{THEORETICAL PERFORMANCE ANALYSIS}

The radius of the blade $(\mathrm{R})$ was varied from $0.3 \mathrm{~m}$ to $2.0 \mathrm{~m}$ and the water head $(\mathrm{H})$ was varied from $3 \mathrm{~m}$ to $5 \mathrm{~m}$. The radius of the nozzle exit area $(\lambda)$ was set at $3 \times 10^{-3} \mathrm{~m}, 4 \times 10^{-3} \mathrm{~m}$ and $5 \times 10^{-3} \mathrm{~m}$, while the nominal diameter of the PVC pipe was varied from a pipe diameter of $1 / 2$ " and 1 ". These were the parameters used for the parametric study in order to investigate the performance of the turbine and to compare it with the experimental results under specified conditions. The theoretical analysis was carried out using the equations described in Parametrial Study based on the range of each parameter.

The operating parameters, such as R, m, H, S and $\omega$, were considered as the predominant parameters that affect the performance of the system. By fixing the other operating parameters, the nomograms, as shown in Figure 3, were designed in order to show the relationship of those parameters with the performance of the system. The nomograms showed the performance curves of the Z-blade turbine under $3 \mathrm{~m}$ and $5 \mathrm{~m}$ of waterhead.

Figure 3 shows that there is a unique turbine diameter, which is also a turning point for the other parameters to significantly change their magnitude and characterize the behaviour of each parameter. At a water head of $5 \mathrm{~m}$, the 0.5 " blade had an optimum rotor diameter of $0.6 \mathrm{~m}$. This was the moment when the turbine rotated at the maximum rotational speed due to the static head together with the pressure exerted by the centrifugal pumping effect. Interestingly, this reaction turbine was capable of rotating at $350 \mathrm{rpm}$, where the mechanical power generated was 63 Watts and it was only consumed at a very low flow rate of $1.48 \mathrm{~L} / \mathrm{sec}$. Meanwhile, the maximum mechanical power generated by the 1 " blade at a water head of $5 \mathrm{~m}$ was $115 \mathrm{~W}$, which was almost double the power generated by the 0.5 " blade. 


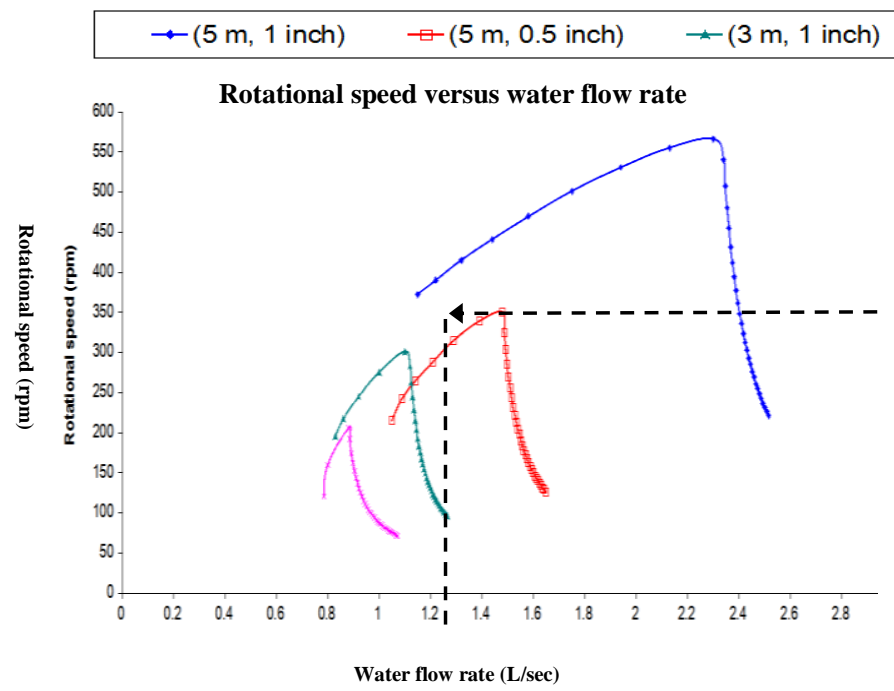

$\rightarrow(3 \mathrm{~m}, 0.5 \mathrm{inch})$
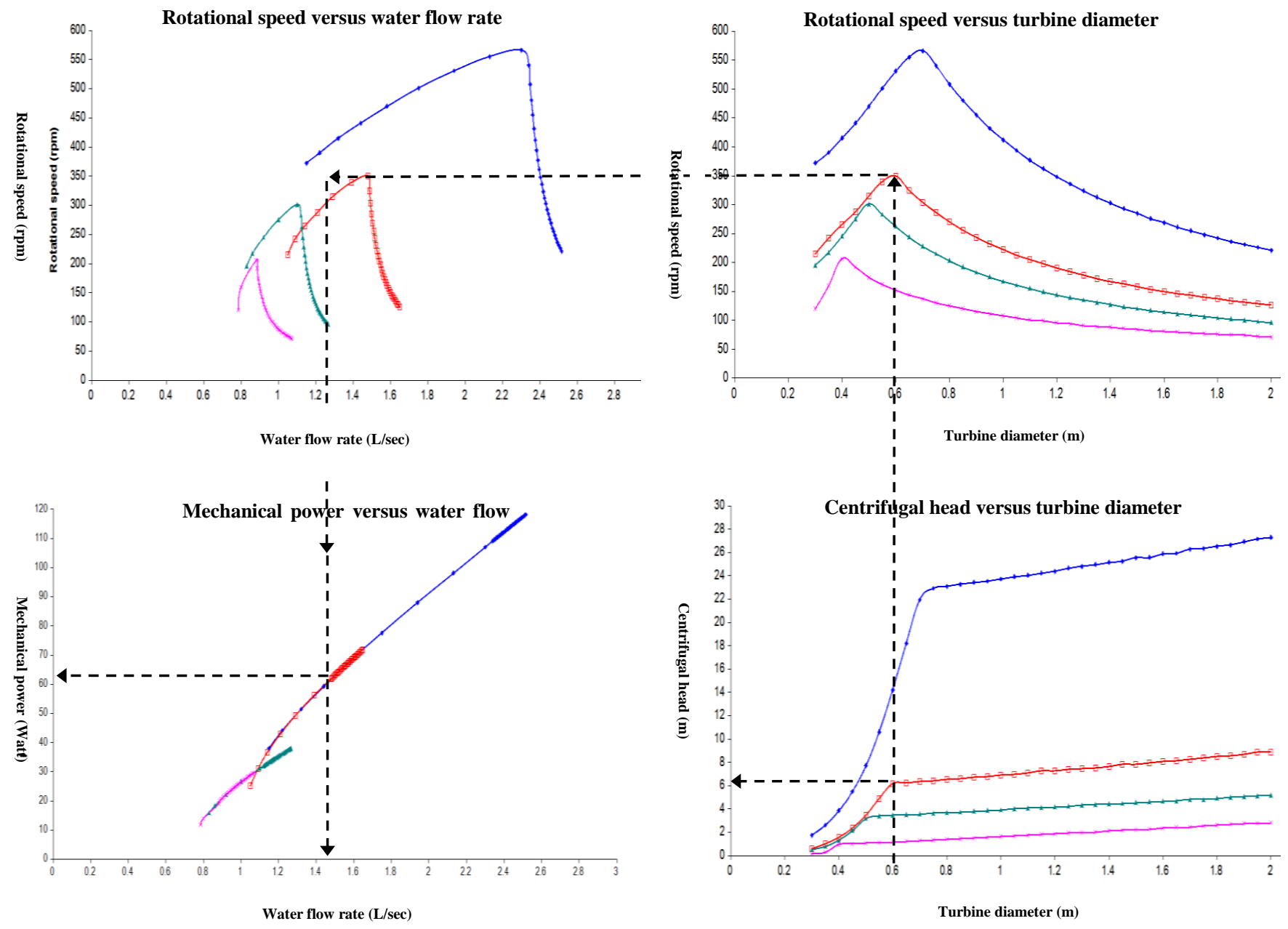

Figure 3. Rotational speed and mechanical power versus water flow rate with rotational speed and centrifugal head versus turbine diameter

It was observed that the optimum turbine diameter and angular velocity shifted to the right with an increase in the water head and PVC pipe size. It should be noted that for any water head and for any pipe size, the Z-blade turbine reaches the highest rotational speed at this unique (optimum) turbine diameter. The optimum diameter is determined at the moment where the rate of increase in the centrifugal head $\left(\mathrm{H}_{\mathrm{c}}\right)$ and the mass flow rate $(\dot{\mathrm{m}})$ drops drastically.

Initially, from a short turbine diameter towards the optimum turbine diameter, the rate of increase in the centrifugal head and rotational speed was high, with an increase in the mass flow rate. Once the turbine reached the maximum speed, the rate of increment of the mass flow rate dropped suddenly and caused the magnitude of the rotational speed to also drop drastically. This occurred due to the rate of increase of the centrifugal head, which was previously high, has been dropped drastically towards the long turbine diameter. Eventually, the rotor speed decreased further as the turbine diameter increased, approaching asymptotically almost half the value of the maximum speed.

There are two components of pressure; one that is derived from the reservoir head $(\rho \mathrm{gH})$ and the other that is associated with the centrifugal pumping action $\left(\mathrm{P}_{\mathrm{c}}={ }^{1} / 2 \rho \omega \mathrm{r}^{2}\right)[3,7,10]$. The combined effect of these pressure components increased the rate of the angular speed of the Z-blade turbine. For the 0.5 " blade with $\mathrm{R}=0.6 \mathrm{~m}$, the centrifugal head, $\mathrm{H}_{\mathrm{c}}$ that was determined was approximately $6 \mathrm{~m}$ even though the waterhead in the reservoir was set at $5 \mathrm{~m}$. Meanwhile, at the same waterhead, the centrifugal pumping effect for the 1" blade was almost five times higher than the supply from the waterhead. It was shown that $\mathrm{H}_{\mathrm{c}}$ was one of the predominant parameters that governed the rate of flow of the water through the turbine, particularly from the short diameter to the optimum turbine diameter. $\mathrm{H}_{\mathrm{c}}$ rapidly increased with increases in the PVC pipe size for a constant waterhead. Besides that, according to Eq. (6), the turbine will not rotate until the pressure from the static head in the reservoir $(\mathrm{pgH})$ consolidates with the pressure associated with the centrifugal pumping action $\left(\mathrm{P}_{\mathrm{c}}={ }_{1}^{1} / 2 \rho \omega \mathrm{r}^{2}\right)[6,9,10]$. The combined effect of these pressures is essential in order to expel the water at a velocity relative to the nozzle. Besides that, it is interesting to note that the effect of the static head on the $\mathrm{H}_{\mathrm{c}}$ diminished specifically at the short turbine diameter.

The mechanical power from the turbine at different water flow rates and turbine diameters are shown in Figure 3. These mechanical power curves were computed using Eq. (8). It can be seen that the mechanical power increased consistently with increases in the mass flow rate, size of pipe and water head. With the turbine diameter ranging from 0.3 
$\mathrm{m}$ to $2 \mathrm{~m}$, the water flow rate for $5 \mathrm{~m}$ of waterhead ranged from $1.05 \mathrm{~L} / \mathrm{sec}$ to $2.51 \mathrm{~L} / \mathrm{sec}$, with a generated power output of up to 115 Watts. Meanwhile, the water flow rate for $3 \mathrm{~m}$ of waterhead ranged from $0.79 \mathrm{~L} / \mathrm{sec}$ to $1.27 \mathrm{~L} / \mathrm{sec}$, with a generated power output of up to 35 Watts. It should be noted that the curve for the $5 \mathrm{~m}$ head was actually a combination of the lines for the 1" blade and 0.5 " blade. It is believed that whatever the size of the pipe used for the turbine blades, it will overlap and connect back in that particular curve as long as the waterhead is consistent.

\section{EXPERIMENTAL PERFORMANCE CHARACTERISTICS}

Consistent results and highly reliable data were obtained by maintaining the experimental procedures and equipment throughout the experimental process. It was found that the experimental performance of the Z-blade turbine was similar in pattern with the theoretical parametric characteristic analysis. Apparently, based on the actual and predicted data, the optimal parameters that mostly influenced the overall turbine performance were identified and discussed.

\section{Relationship between Rotational Speed, Turbine Diameter and Flow Rate}

Figure 4 shows the experimental and theoretical curves for the Z-blade reaction water turbine with the rotor diameter varying from $0.3 \mathrm{~m}$ to $2.0 \mathrm{~m}$ at different waterheads ranging from $3 \mathrm{~m}$ to $5 \mathrm{~m}$. The Z-blade turbine had one nozzle for each blade arm, with the nozzle diameter set at $0.008 \mathrm{~m}$. The turbine was developed using grey PVC pipes sized at 1 " (nominal diameter $=0.025 \mathrm{~m}$ ) and $1 / 2$ " (nominal diameter $=0.015 \mathrm{~m}$ ).

As shown in Figure 4, for a constant operating head $(\mathrm{H})$, there is a unique rotor diameter where the turbine has the highest maximum angular speed $(\omega)$, and this is taken as the optimum turbine diameter. The optimum diameter varies linearly with the water head $(\mathrm{H})$, either for the theoretical or experimental data. In addition, the water flow rate $(\dot{\mathrm{m}})$ through the turbine increases with increments in the waterhead $(\mathrm{H})$.

It can be seen in Figure 4 that from the short diameter until the optimum turbine diameter, the rotational speed and water flow rate increased as the turbine diameter increased. Overall these three parameters $(\omega, \dot{m}$, and optimum diameter) increased substantially in proportion to increases in the waterhead. Initially, when the turbine diameter was small, the rotor speed increased rapidly. This was due to the effect of the mass flow rate (m) and the static head, H. This static head was produced due to the physical difference between the water level in the water tank and the position of the turbine [6, 9]. In fact, apart from the static head, the Z-blade turbine was also subjected to pressure from the centrifugal head $\left(\mathrm{H}_{\mathrm{c}}\right)$. This centrifugal pumping action was created due to the increment in the turbine diameter and the rotational speed $(\omega)$ of the turbine as it approached the maximum rotational speed.

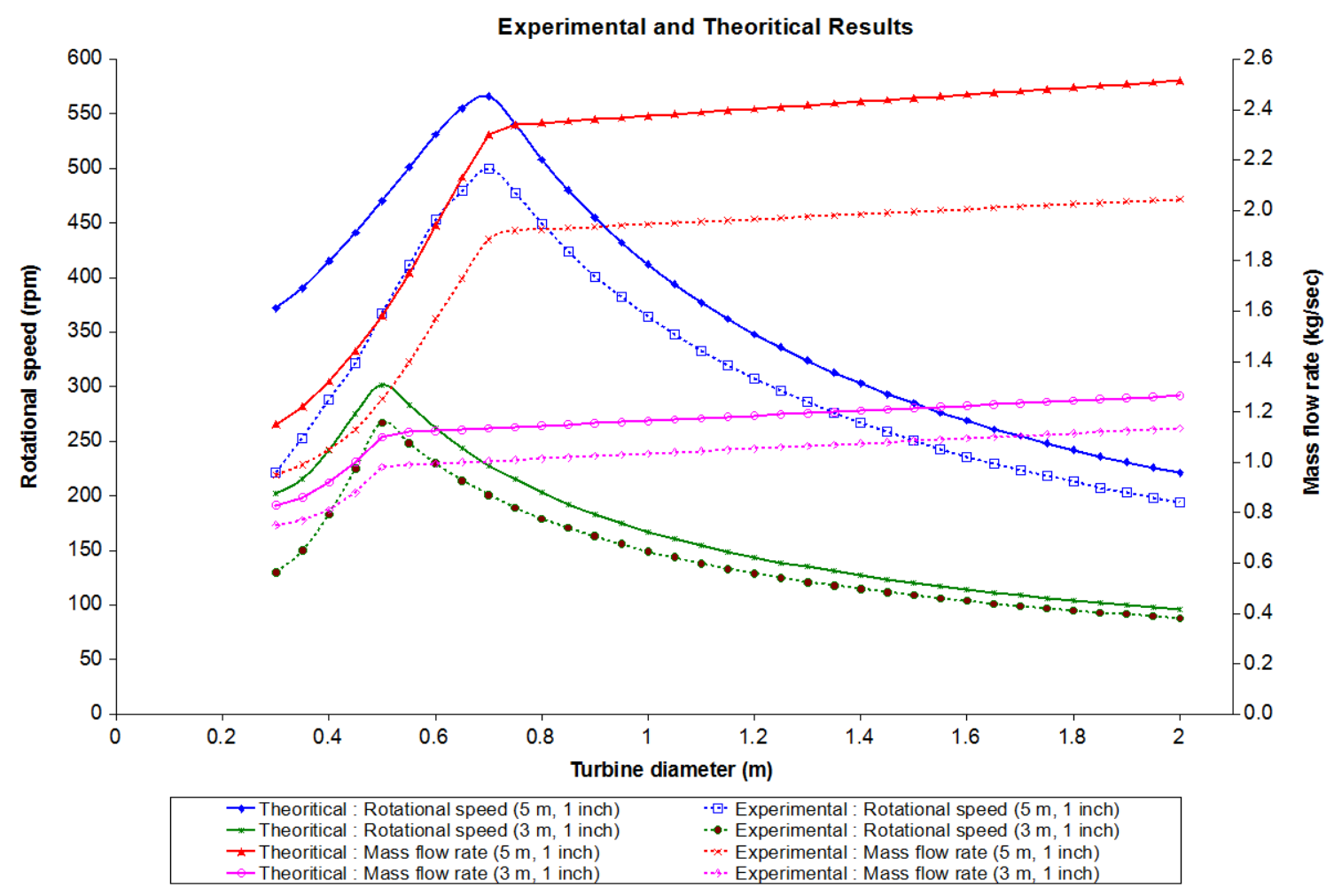

Figure 4. Experimental and theoretical results for 1" pipe

As shown in Figure 3, once the rotor reached the maximum speed, the increase in the rate of flow of the water through the turbine started to become extremely low, even though the diameter the of Z-blade had increased, unlike at the initial 
stage where the mass flow rate increased substantially. Even though the magnitude of the mass flow rate and centrifugal pumping head had considerably small increments towards the long turbine diameter, the rotational speed reduced gradually due to the effect of the increased blade length.

From the experimental data shown in Figure 4, when the height of the potential energy was at $5 \mathrm{~m}$, the turbine reached the maximum speed of $500 \mathrm{rpm}$ by only requiring a water flow rate of $1.89 \mathrm{~L} / \mathrm{sec}$. This speed differed slightly by about $11.7 \%$ from the theoretical estimation, where the calculated maximum speed was $566 \mathrm{rpm}$. It should be noted that the Zblade turbine only requires low-head and low-flow water resources, which is very different compared to other simple reaction hydro turbines.

\section{Effect of Nozzle Exit Area on Turbine Performance}

In Figure 5 indicates the varying actual turbine diameter of the turbine rotation and water flow rate of $4 \mathrm{~m}$ waterhead. The curve designs for velocity and flow rate for the three nozzle diameters are similar. The flow rate however increased when the output area of the piston was increased.

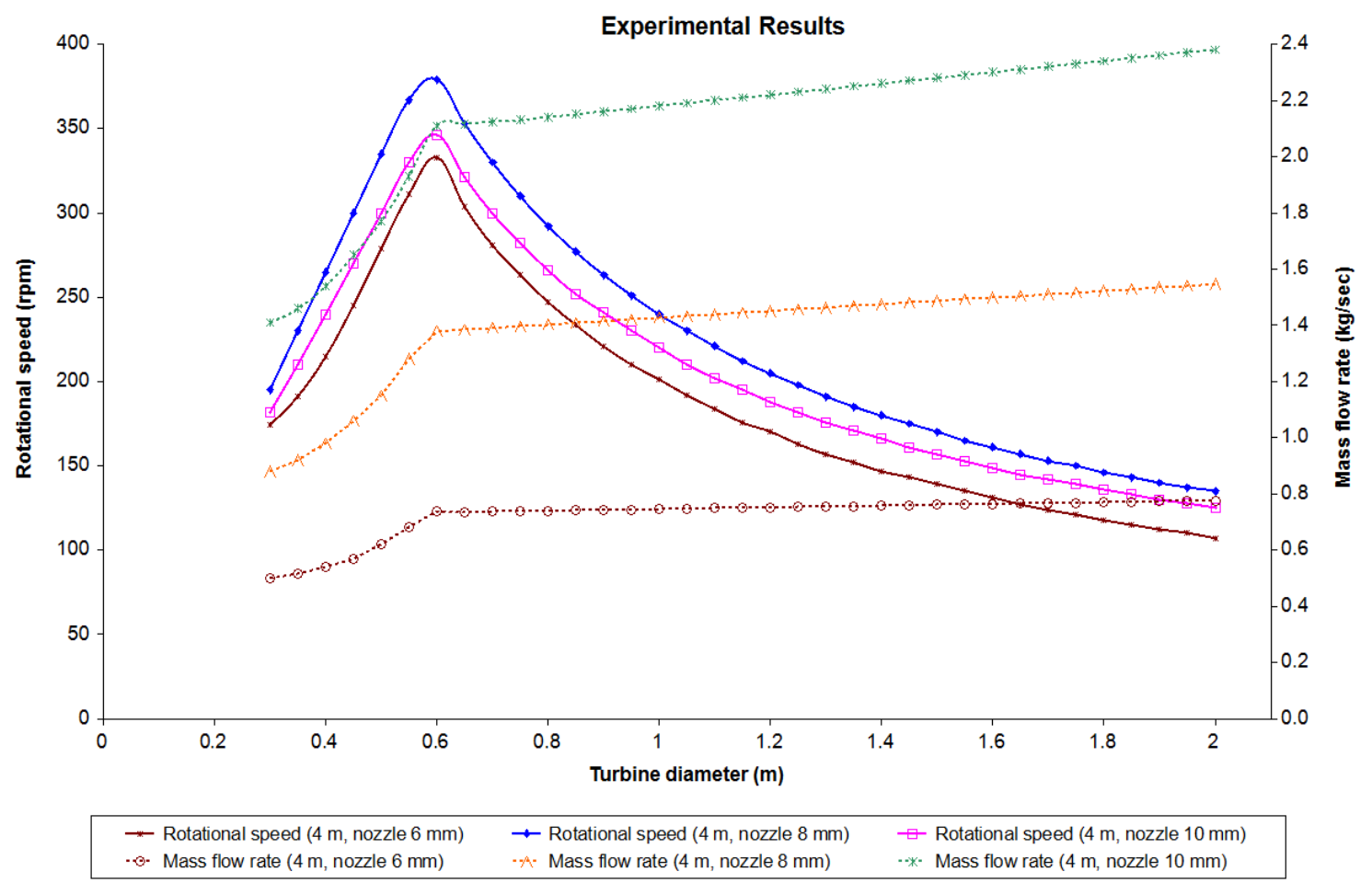

Figure 5. Variation of speed with turbine diameter for several nozzle exit areas

The $0.008 \mathrm{~m}(\mathrm{~A}=1.0 \times 10-4 \mathrm{~m})$ turbine with a nozzle diameter was greater compared to $0.01 \mathrm{~m}(\mathrm{~A}=157.1 \times 10-6 \mathrm{~m})$ and $0.006 \mathrm{~m}(\mathrm{~A}=56.6 \times 10-6 \mathrm{~m})$ in nozzle diameter. While the turbine with a nozzle diameter of 0.01 was the largest mass flow rate, followed by $0.008 \mathrm{~m}$ and $0.006 \mathrm{~m}$, the turbine with a nozzle diameter of $0.008 \mathrm{~m}$ was at the highest rotational speed reported.

As Eq. (7) shows, the water flow rate component $\left(\frac{\dot{m}}{\rho A}\right)$ and the total exit area of the nozzle highly affect the angular velocity of the rotor provided that the diameter and waterhead of the turbine are fixed. However, the mass flow rate remained the key parameter in deciding the output of the Z-blade turbine in circumstances where the nozzle exit area remained unchanged.

\section{CONCLUSIONS}

The performance characteristics of a simple reaction turbine, known as a Z-blade turbine, have been presented by means of nomograms and curves. The Z-Blade turbine has been examined and has shown good potential to be used for low waterhead ( $3 \mathrm{~m}, 4 \mathrm{~m}$ and $5 \mathrm{~m}$ ) and low water flow rate (less than $2.5 \mathrm{~L} / \mathrm{sec}$ ).

For any waterhead and for any pipe size, there is a unique turbine length, called the optimum diameter, at which the Z-blade turbine reaches the maximum rotational speed. Furthermore, at this point, the increase in rate in the centrifugal head $\left(\mathrm{H}_{\mathrm{c}}\right)$ and the mass flow rate $(\dot{\mathrm{m}})$ begin to drop drastically. It is the turning point for other parameters to change their magnitude significantly and simultaneously, thus characterizing the behaviour of each parameter.

Besides that, for any size of the nozzle exit area, the curve patterns of the speed and flow rate are similar, except for the magnitude. The bigger the nozzle diameter, the higher will be the quantity of water required by the turbine. However, 
having a nozzle with a large diameter does not guarantee that the turbine will rotate very fast. This is because the component, $\left(\frac{\dot{m}}{\rho A}\right)$, will play a key role in determining the speed of the turbine.

In general, these parameters $\left(\omega, \mathrm{H}_{\mathrm{c}}, \dot{\mathrm{m}}\right.$ and optimum diameter) have been substantially increased in proportion to the increment in the waterhead. There are two components of the water pressure, namely the static head in the reservoir $(\mathrm{H})$ and the centrifugal head $\left(\mathrm{H}_{\mathrm{c}}\right)$ which are associated with the centrifugal pumping action. The magnitude of $\mathrm{H}_{\mathrm{c}}$ increases with increments in the rotational speed, and simultaneously increases the mass flow rate. It should be noted that the turbine will only rotate when the pressure from the waterhead consolidates with the pressure associated with the centrifugal pumping action. These combined effects are essential in order to expel water at a velocity relative to the nozzle.

Based on the actual performance curves and nomogramming, it is shown to be realistic for the water head, rotor length, pipeline size and nozzle exit area as well as the weight flow rate, centrifuge head, power output and efficiency. Finding out best output of Z-Blade turbine are the turbine diameter, mass flow rate and a water head.

\section{ACKNOWLEDGMENTS}

The authors would like to thank the Ministry of Higher Education, Malaysia for funding this research (MTUNC/2019/FTKEE-CERIA/MC0013), and Universiti Teknikal Malaysia Melaka (UTeM) and Universiti Kebangsaan Malaysia (UKM) for providing the laboratory facilities and technical support.

\section{REFERENCES}

[1] I. Safdar, S. Sultan, H. A. Raza, M. Umer, and M. Ali, "Empirical analysis of turbine and generator efficiency of a pico hydro system,” Sustain. Energy Technol. Assessments, vol. 37, no. August 2019, p. 100605, 2020, doi: 10.1016/j.seta.2019.100605.

[2] B. N. Fortaleza, R. O. Serfa Juan, and L. K. S. Tolentino, "IoT-based pico-hydro power generation system using pelton turbine," J. Telecommun. Electron. Comput. Eng., vol. 10, no. 1-4, pp. 189-192, 2018.

[3] A. Lahimer et. al, "Research and development aspects of pico-hydro power," Renewable and Sustainable Energy Reviews, vol. 16, no. 8, pp. 5861-5878, 2012.

[4] S. J. Williamson, B. H. Stark, and J. D. Booker, "Low head pico hydro turbine selection using a multi-criteria analysis," Renew. Energy, vol. 61, pp. 43-50, Jan. 2014, doi: 10.1016/j.renene.2012.06.020.

[5] K. Kusakana, "A survey of innovative technologies increasing the viability of micro-hydropower as a cost effective rural electrification option in South Africa," Renew. Sustain. Energy Rev., vol. 37, pp. 370-379, 2014, doi: 10.1016/j.rser.2014.05.026.

[6] A. Date, A. Date, and A. Akbarzadeh, "Investigating the potential for using a simple water reaction turbine for power production from low head hydro resources," Energy Conversion and Management, vol. 66. pp. 257-270, 2013, doi: 10.1016/j.enconman.2012.09.032.

[7] A. Akbarzadeh, C. Dixon, and P. Johnson, "Parametric analysis of a simple reaction water turbine and its application for power production from low head reservoirs," Proceedings of the ASME Fluids Engineering Division Summer Meeting, vol. 2. pp. 697-706, 2003.

[8] A. Date, "Low head simple reaction water turbine," Thesis Sch. Aerosp. Mech. Manuf. Eng. RMIT Univ., no. April, pp. 1-251, 2009.

[9] A. Date and A. Akbarzadeh, "Design and analysis of a split reaction water turbine," Renew. Energy, vol. 35, no. 9, pp. 19471955, 2010, doi: 10.1016/j.renene.2010.01.023.

[10] A. Date and A. Akbarzadeh, "Design and cost analysis of low head simple reaction hydro turbine for remote area power supply," Renewable Energy, vol. 34, no. 2. pp. 409-415, 2009, doi: 10.1016/j.renene.2008.05.012. 